'División de Pediatría, Pontificia Universidad Catolica de Chile. Santiago, Chile. ${ }^{2}$ Servicio de Pediatría Complejo Asistencial Dr. Sótero del Río. Santiago, Chile. aResidente de Endocrinología Pediátrica.

Este trabajo no contó con financiamiento.

Recibido el 4 de noviembre de 2014, aceptado el 25 de abril de 2015.

Correspondencia a:

Dra. Carolina Mendoza F. División de Pediatría. Santiago, Chile. Lira $85,5^{\text {to }}$ piso.

Fax: 56-2-26384307 camendoza@uc.cl caromendozafuentes@gmail.com

\section{Orbitopatía de Graves en pediatría}

\author{
CAROLINA MENDOZA F. ${ }^{1, a}$, PATRICIA LACOURT R. ${ }^{2}$ \\ Graves' orbitopathy in pediatrics
}

Graves' orbitopathy $(G O)$ is rare in pediatric patients, however is the most common extrathyroid manifestation of Graves' disease (GD), being present in $30-67 \%$ of patients. GO is an autoimmune inflammatory disorder involving orbital connective and fatty tissues as well as the extraocular muscles. In children, $G O$ is less common and less severe than in adults. The most common symptoms are upper eyelid retraction, conjunctival injection, and proptosis and periorbital edema. Severe complications include dysthyroid optic neuropathy, corneal ulceration and eyeball subluxation. The diagnosis is established by clinical, laboratory and imaging findings. There are no management guidelines for $G O$ in children but adult recommendations include the assessment of clinical activity and its severity, to implement the best treatment. Supportive therapies are intended to relieve symptoms and prevent corneal damage in mild cases. Tobacco exposure should also be avoided. The first line of treatment is systemic administration of corticosteroids in active and severe cases. Other options as somatostatin use and retrobulbar radiation have not been used in children, therefore their use is not recommended.

(Rev Med Chile 2015; 143: 1034-1041)

Key words: Child; Graves disease; Graves ophthalmopathy; Glucocorticoids.
$\mathrm{E}$ 1 hipertiroidismo autoinmune es la causa más común de tirotoxicosis en niños y adolescentes. La incidencia reportada en Europa es de 0,8/100.000 niños/año, con una relación por sexo femenino: masculino de 3-5:1 ${ }^{1}$. La Orbitopatía de Graves (OG) es la manifestación extratiroidea más frecuente de la enfermedad de Graves (EG) y se presenta en 30-67\% de los pacientes. Es una patología poco frecuente en pediatría y el riesgo de desarrollarla aumenta con la edad ${ }^{2}$. Al comparar por grupos etarios se observa una frecuencia de $31,8 \%$ en pacientes menores de 11 años versus $68,2 \%$ en adolescentes (11-18 años) ${ }^{3}$.

La OG es una enfermedad inflamatoria autoinmune. Se asocia a hipertiroidismo autoinmune en $80-90 \%$ de los casos, 3-4\% de los casos se presenta en pacientes con tiroiditis de Hashimoto y $5-10 \%$ en pacientes eutiroideos ${ }^{4}$. El $80 \%$ de los casos de OG se presenta dentro de los primeros 18 meses desde que se diagnostica la enfermedad tiroidea, sin embargo, se puede desarrollar antes o después de la aparición del hipertiroidismo ${ }^{5}$.

\section{Fisiopatología}

La patogenia de la OG está determinada por una infiltración perivascular difusa de linfocitos T CD4+, CD8+, linfocitos B, células plasmáticas y macrófagos; estas células liberan citoquinas inflamatorias y prostaglandinas las que inducen remodelación del tejido conectivo, lo que asociado a una infiltración de glicosaminoglicanos producen un aumento de volumen de los músculos extraoculares $(\mathrm{MExO})$ y del tejido adiposo orbitario ${ }^{6-7}$.

\section{Fibroblasto orbitario (FO): la célula diana}

Actualmente se reconoce a los FO como las células diana en la $\mathrm{OG}^{7-8}$. Estudios sugieren que los linfocitos T CD8+ actuarían reconociendo a los FO y estimulando su proliferación vía complejo mayor de histocompatibilidad clase II. A diferencia de las células de los $\mathrm{MExO}$, los FO expresan antígeno leucocitario humano (HLA-DR), los que actuarían como células presentadoras de antígenos ${ }^{9}$. 
Los FO se clasifican en base a la presencia o ausencia de una glicoproteína de superficie CD90, conocida como antígeno timocito-1 (Thy-1), este antígeno jugaría un rol esencial en la respuesta inmune $e^{10-11}$. En respuesta a la liberación de citoquinas, como interferón- $\gamma$ y factor de necrosis tumoral (TNF), los FO Thy-1 (+), aumentan la producción de ácido hialurónico $(\mathrm{AH})^{12}$, mientras que el grupo de FO Thy-1(-) o preadipocitos se diferencian a adipocitos, aumentando el volumen del tejido conectivo orbitario. Este proceso de adipogénesis se asocia a un aumento en la expresión del receptor de TSH (TSH-R) y contribuye en estadíos posteriores de la OG a una mayor producción de grasa orbitaria y exoftalmos.

La inflamación característica de los $\mathrm{MExO}$ y del tejido conectivo/graso orbitario es secundaria a edema y acumulación de glicosaminoglicanos (GAG), compuestos hidrofílicos que osmóticamente atraen agua. Se ha comprobado que el contenido de GAG, especialmente condroitín sulfato y ácido hialurónico en la órbita de pacientes con OG es $70 \%$ mayor que en sujetos control ${ }^{13}$.

\section{TSH-R como autoantígeno}

En la EG, el TSH-R de las células foliculares tiroideas actúa como autoantígeno y los anticuerpos dirigidos contra su superficie estimulan la sobreproducción de hormonas tiroideas. En la década de los 90 se detectó por primera vez la presencia del TSH-R en FO, hallazgo que fue confirmado posteriormente ${ }^{14-15}$. Estudios clínicos sugieren que este receptor actúa como autoantígeno en la OG, hecho que queda demostrado al detectar anticuerpos dirigidos contra el TSH-R (TRAb) en casi todos los pacientes con OG, incluidos aquellos eutiroideos y que los niveles de la fracción estimulante de los TRAb se correlacionan con la severidad y nivel de actividad de la enfermedad ${ }^{16-17}$, además la unión de los TRAb al TSH-R en los FO produce una regulación positiva en la sintasa de ácido hialurónico determinando una producción excesiva de AH.

\section{Factores de riesgo}

\section{Género y raza}

$\mathrm{Al}$ igual que otras enfermedades autoinmunes, la OG es más frecuente en mujeres que en hombres, en una razón de 2:1, sin embargo, los hombres presentan formas más severas con mayor riesgo a mayor edad. Se ha observado que pacientes de raza asiática tienen menor probabilidad de desarrollar la enfermedad que pacientes europeos ${ }^{21}$.

\section{Factores genéticos}

Los estudios genéticos se han enfocado en polimorfismos de inmunomoduladores incluyendo; HLA-DR3, CTLA-4, IL-1, IL-23R, CD40, TNF-b, gen del TSHR ${ }^{21}$, pero los resultados han sido contradictorios y no han logrado demostrar una base genética clara que explique la $\mathrm{OG}^{22}$.

\section{Tabaco}

El consumo de tabaco es el factor de riesgo más importante para el desarrollo y progresión de la OG. Entre los pacientes con EG, los fumadores tienen mayor riesgo de desarrollar OG (OR 7,7 vs 1,7$)$ y de presentar formas más severas ${ }^{23}$. La respuesta al tratamiento no sólo es menor, incluso es más lenta en fumadores versus no fumadores ${ }^{24}$. Existe evidencia que no sólo el tabaquismo activo, sino que también el tabaquismo pasivo tienen impacto en el desarrollo y progresión de la OG. Krassas et $\mathrm{al}^{3}$ agruparon países según la prevalencia de tabaquismo en adolescentes, encontrándose $36 \%$ de OG en países con una prevalencia de consumo de tabaco mayor al $25 \%$ en comparación a $25,9 \%$ en aquellos países con menor prevalencia de tabaquismo. En este mismo estudio se evidenció que en los países con alta prevalencia de tabaquismo, la distribución de OG era igual para mayores y menores de 11 años, lo que hace suponer que esto se debe a la exposición pasiva al tabaco en los pacientes de menor edad.

Los mecanismos por lo cuales el tabaco afecta la OG no están del todo claros, pero estudios in vitro han mostrado que residuos de cigarro y la hipoxia secundaria aumentan la producción de GAG, además de aumentar la adipogénesis por parte de los $\mathrm{FO}^{25}$.

\section{Disfunción tiroidea}

Tanto el hipertiroidismo como el hipotiroidismo tienen efectos negativos en el curso de la OG. Estudios en pacientes adultos, muestran una mayor proporción de hipertiroidismo no controlado en pacientes con OG moderada-severa en comparación a pacientes con enfermedad leve. También se ha demostrado que posterior a tratamiento con radioyodo, en pacientes en los que se esperó tener documentado un hipotiroidismo para iniciar terapia con levotiroxina, el riesgo 
relativo de desarrollar o empeorar la OG fue de 1,64 (95\% CI: 1,1-2,6) ${ }^{26}$. Una explicación posible es que ambos estados se asocian a activación del TSH-R, tanto por su estimulador nativo, la TSH como por los TRAbs, los que producen un aumento en la expresión de antígenos tiroideos y una exacerbación de las reacciones inmunes dirigidas contra los antígenos de la órbita ${ }^{22}$. Por esta razón, restaurar y mantener un estado eutiroideo es fundamental en pacientes con EG, especialmente en aquellos con $\mathrm{OG}^{27}$. Sin embargo, en estudios en población pediátrica ${ }^{1,34}$ no se ha logrado remisión de los signos de OG a pesar de restaurar un estado eutiroideo, la explicación de esta respuesta disímil no ha sido aclarada.

\section{Radioyodo}

La terapia con radioyodo se ha asociado a un mayor riesgo de progresión y ocurrencia de novo de OG, especialmente en fumadores ${ }^{28}$. Un metanálisis ${ }^{29}$, que incluye pacientes adolescentes, mostró que el radioyodo tiene un efecto adverso sobre la OG en $15-20 \%$ de los casos, no obstante este riesgo puede disminuirse con el uso de glucocorticoides profilácticos $^{30}$. El mecanismo por el cual esto ocurre es probablemente una exacerbación de la respuesta autoinmune, debido a la liberación brusca de antígenos, secundaria a la acción citolítica del radioyodo en las células foliculares del tiroides.

\section{Manifestaciones clínicas (Tabla 1)}

La OG se presenta con menor frecuencia y de forma menos severa en niños que en pacientes adultos $^{31-32}$. Los síntomas más comunes son: retracción de párpados superiores, proptosis, edema-eritema de tejidos periorbitarios y de la conjuntiva. Estos síntomas son característicos de la OG en niños; mientras que las manifestaciones más severas como el estrabismo restrictivo, la neuropatía óptica distiroidea (NOD) y la quetaropatía por exposición son típicas del adulto. En una serie ${ }^{33}$ de 120 pacientes adultos el estrabismo restrictivo alcanzó $42,5 \%$ y la NOD 5,8\%, en comparación a una serie pediátrica ${ }^{34}$ donde los porcentajes fueron $1,2 \%$ y $0 \%$ respectivamente. Holt et $\mathrm{al}^{36}$ describieron la presentación clínica en pacientes prepuberales quienes exhibieron características típicas descritas en niños con OG, mientras que en el grupo postpuberal 5 pacientes presentaron estrabismo restrictivo. En pacientes pediátricos $\operatorname{chilenos}^{37}$, no se encontraron diferencias por edad en la forma de presentación clínica de la OG. Una hipótesis que podría explicar la diferencia en severidad entre niños y adultos es la exposición al tabaco, ya que las manifestaciones clínicas de OG en pacientes pediátricos se asemejan a las del adulto cuando los pacientes entran en etapa de adolescencia, grupo en el cual la prevalencia de consumo de tabaco aumenta.

\section{Diagnóstico}

\section{Evaluación clínica}

Todos los pacientes con EG deben ser derivados a oftalmología para realizar mediciones específicas de agudeza visual, evaluación con lámpara

Tabla 1. Características clínicas orbitopatía de Graves

\begin{tabular}{|ll|}
\hline Síntomas & Signos \\
\hline Sensación de cuerpo extraño $37 \%$ & Retracción palpebral $83 \%$ \\
\hline Fotofobia $28,6 \%$ & Exoftalmos $74 \%$ \\
\hline Dolor o discomfort $25,7 \%$ & Lid lag $74 \%$ \\
\hline Diplopía $17 \%$ & Inyección conjuntival $49 \%$ \\
\hline Epífora $8,6 \%$ & Resistencia a retropulsión $46 \%$ \\
Disminución de agudeza visual $5,7 \%$ & Lagoftalmos $37 \%$ \\
& Manchas corneales $34 \%$ \\
& Quemosis $23 \%$ \\
& Queratitis límbica $17 \%$ \\
& Alteración motilidad músculos extraoculares $11 \%$ \\
\hline
\end{tabular}

Vikram $D^{35}$ (35 pacientes en tres centros USA). 
de hendidura, presión intraocular y cuantificación de proptosis, esto permite realizar un diagnóstico precoz e instaurar medidas terapéuticas para evitar complicaciones. Más adelante se discute sobre actividad y severidad clínica.

\section{Exámenes de laboratorio}

Están orientados a evaluar el estado de función tiroidea. En el estudio de Krassas ${ }^{3}$ los exámenes más frecuentemente solicitados fueron: TSH (100\%), T4 libre (94\%), TRAb (88\%), luego anticuerpos antiperoxidasa y T3 libre (60\%).

\section{Imágenes}

La tomografía computada (CT), resonancia magnética (MRI) y ultrasonografía (US) son útiles en el diagnóstico y seguimiento clínico-quirúrgico, permitiendo diferenciar el compromiso inflamatorio muscular agudo de los estadíos crónicos con presencia de fibrosis ${ }^{38}$. Tanto la CT y MRI establecen el grado de compromiso de los $\mathrm{MExO}$ y de la grasa orbitaria, permiten excluir otras patologías orbitarias (especialmente en casos de proptosis unilateral) y planificar cirugía. La CT es el examen de elección para el diagnóstico de $\mathrm{OG}^{39}$, es un examen de menor costo y con mayor accesibilidad que la MRI. Se recomienda realizarla antes y después de la cirugía para definir el sitio y extensión de la descompresión ósea ${ }^{40}$. Su desventaja son la exposición a radiación y menor capacidad para identificar cambios en tejidos blandos que son importantes al momento de evaluar el grado de actividad de la enfermedad y de respuesta a tratamiento. La US es una herramienta de bajo costo, sin exposición a radiación, sin embargo, otorga menos información sobre los $\mathrm{MExO}$ y las paredes orbitarias, además tiene una alta variabilidad intra e interobservador. Otra técnica disponible es la cintigrafía con octreótide (octreoscan), la cual tendría utilidad en pacientes con enfermedad activa, sin embargo, es una técnica costosa, no específica y con alta exposición a radiación, lo que restringe su utilidad clínica.

Después de realizada la evaluación de un paciente con OG se debe definir el tratamiento óptimo, para eso es necesario conocer el nivel de actividad y severidad de la enfermedad. La severidad $^{41}$ se refiere al espectro de manifestaciones clínicas, que van desde signos leves como edema, eritema, retracción de párpados y proptosis hasta signos severos como compromiso de los $\mathrm{MExO}$, restricción en la motilidad ocular y ocasionalmente pérdida de visión en casos de NOD. La actividad clínica tiene relación con el curso bifásico que sigue la enfermedad, con una etapa activa que dura entre 6 a 18 meses, seguida por una fase inactiva o estable.

El año 2006 se introdujo la clasificación VISA ${ }^{42}$ a la práctica clínica y ha sido adoptada por la International Thyroid Eye Disease Society (ITEDS) para registrar información y para planificar el manejo. Este registro permite graduar tanto la severidad clínica como la actividad de la enfermedad. Divide las características clínicas en 4 parámetros: V (visión, NOD); I (inflamación, congestión); S (estrabismo, restricción motilidad); A (apariencia, exposición). Se realiza un registro en cada visita, sumándose el grado de severidad y progreso para cada uno de estos 4 parámetros. El grado de actividad se determina en base al deterioro en cualquiera de estos puntos. Este formulario se encuentra disponible para descarga gratuita en el sito web de la ITEDS: www.thyroideyedisease.org.

Es importante recordar que la evaluación clínica debe incluir una evaluación de calidad de vida (QoL), ya que se ha observado un deterioro de ésta en pacientes con OG. Entre los factores que contribuyen a este deterioro están: alteraciones en la función visual, discomfort ocular y alteraciones en la apariencia física, los que producen una peor autoimagen, problemas de sueño y mal rendimiento social y laboral ${ }^{43}$. Existe un instrumento de evaluación de calidad de vida enfermedad específico validado, llamado GO-QoL ${ }^{44}$, herramienta que puede ser usada para medición de outcomes primarios en ensayos clínicos.

\section{Complicaciones}

La OG en niños es menos severa y presenta menor frecuencia de complicaciones que en los adultos. Las complicaciones incluyen tanto secuelas estéticas como funcionales, entre éstas están ${ }^{43}:$ 1) Neuropatía óptica distiroidea (DON): complicación severa que afecta a 5\% de los pacientes adultos con OG, puede producir ceguera y se presenta como un deterioro brusco de la visión o cambios en la intensidad o calidad de la visión de colores; 2) Compromiso corneal: queratitis por exposición o úlceras corneales, se presenta en pacientes que tienen exposición permanente de la córnea en relación a la retracción palpebral y al 
grado de proptosis; 3) Subluxación globo ocular: es un desplazamiento parcial del globo ocular desde la órbita, por delante de los párpados, se asocia a dolor y compresión del nervio óptico. Ante la sospecha de cualquiera de estas complicaciones se debe derivar de manera urgente a oftalmólogo.

\section{Manejo}

No existen guías publicadas para el manejo de la OG en pediatría, sin embargo, el Grupo Europeo de Orbitopatía de Graves (EUGOGO) ${ }^{27}$ entrega algunas recomendaciones. En este grupo de pacientes el énfasis está en lograr y mantener un estado eutiroideo, evitar la exposición al tabaco y el tratamiento, en la mayoría de los casos, es conservador. En la Tabla 2 se muestran las recomendaciones con su nivel de evidencia.

En la encuesta de Krassas ${ }^{3}$ el tratamiento de elección para pacientes con OG fue conservador, con una política de "esperar y observar" en 70\% de los encuestados. Un $28 \%$ de los encuestados recomendó usar corticoides sistémicos en casos de deterioro clínico o ausencia de mejoría cuando el paciente se encuentra eutiroideo. El uso de otras alternativas terapéuticas fue escaso, sólo 1,5\% recomendó utilizar análogos de somatostatina y ninguno de los encuestados recomendó la radioterapia retrobulbar.

La clasificación VISA ordena las cuatro funciones alteradas en orden descendente según prioridad para tratamiento ${ }^{41}$.

\section{Medidas generales}

Destinadas a aliviar síntomas y prevenir el deterioro de la OG. Las gotas lubricantes y ungüentos ayudan a proteger la integridad de la córnea, este es el manejo recomendado en los casos de OG leve.

\section{Tabla 2. Recomendación EUGOGO en el manejo de la OG en pediatría}

\footnotetext{
Restablecer y mantener eutiroidismo precozmente (III,B)

Niños con OG deben ser manejados de manera conservadora si no existe riesgo visual (IV,C)

Utilizar medidas generales para tratar síntomas (IV,C)

Se debe evitar uso de corticoides en pediatría (IV,C)

Radioterapia orbitaria está contraindicada en niños (IV,C)

Evitar la exposición activa y pasiva al tabaco (IV,C)
}

Otra medida fundamental es el cese del tabaquismo activo y del pasivo en los casos de pacientes pediátricos. Es primordial instaurar de manera precoz tratamiento médico para el manejo del hipertiroidismo, ya que se ha visto que el curso de la enfermedad es mejor en los pacientes cuando se restablece el eutiroidismo ${ }^{41}$, en pediatría el tratamiento de elección para lograr este objetivo son las drogas antitiroideas.

\section{Glucocorticoides (GC)}

El objetivo de la terapia inmunosupresora es disminuir la inflamación y congestión del tejido orbitario y así prevenir la progresión de la enfermedad autoinmune ${ }^{45,46}$, sólo los pacientes en estadío activo responden a esta terapia. Según la recomendación EUGOGO ${ }^{27}$ los GC son el tratamiento de elección en OG moderada y severa en etapa activa. En adultos ${ }^{47,48}$ se ha demostrado que la administración endovenosa es más efectiva que la administración vía oral, ya sea como monoterapia o combinada con radioterapia, con una dosis acumulativa recomendada no mayor a $8 \mathrm{~g}$. Un estudio reciente ${ }^{49}$ en adultos, mostró que un protocolo de administración semanal de metilprednisolona endovenosa era más eficiente y seguro que un protocolo diario para pacientes con OG activa moderada a severa. En pediatría no existen estudios randomizados pero se han sugerido ${ }^{50,51}$ dosis entre 5-20 mg de prednisona al día vía oral por 4-6 semanas, seguido de una disminución progresiva de la dosis, teniendo en consideración los efectos adversos asociados al uso prolongado de glucocorticoides. Otra indicación para el uso de $\mathrm{GC}$ es en pacientes fumadores, aquellos que tienen OG activa o con hipertiroidismo severo, en los cuales los GC se utilizan de manera profiláctica para prevenir la exacerbación de la OG asociada al uso de radioyodo.

\section{Radioterapia orbitaria}

Es una terapia segura y bien tolerada en adul$\operatorname{tos}^{52}$, con buenos resultados cuando se utiliza en combinación con GC vía oral en casos de OG activa moderada-severa. Esta terapia no está recomendada en pacientes menores de 35 años por el riesgo carcinogénico.

\section{Análogos de somatostatina}

La presencia de receptores de somatostatina en los tejidos retrobulbares, asociado a los efectos 
inhibitorios del octreótide en la función inmune y en el crecimiento de fibroblastos, proveen una base teórica para su uso en el tratamiento de la $\mathrm{OG}^{50}$. Sin embargo, estudios randomizados muestran escaso o nulo beneficio, por lo que no se recomienda su uso.

\section{Terapias biológicas}

El uso de Rituximab ${ }^{\circledR}$ ha sido evaluado en pacientes adultos como una alternativa terapéutica en casos de OG activa severa en pacientes que tienen contraindicación o una respuesta inadecuada al uso de GC. Sin embargo, una revisión Cochrane del año 2013 concluyó que la evidencia es insuficiente para apoyar su uso en OG.

\section{Cirugía}

En los casos activos la descompresión orbitaria tiene utilidad cuando falla la terapia médica o ante la amenaza de pérdida visual como ocurre en los casos de $\mathrm{NOD}^{27}$. En pacientes que presentan al menos 6 meses de inactividad clínica la cirugía permite corregir las manifestaciones residuales o estéticas.

\section{Conclusiones}

La OG es una enfermedad inflamatoria autoinmune, determinada por la remodelación del tejido conectivo y adiposo periorbitario, asociada a una infiltración de glicosaminoglicanos. Es una patología poco frecuente en pediatría, probablemente debido a la baja incidencia de EG en este grupo etario. Los pacientes pediátricos tienen el mismo riesgo de desarrollar OG que los adultos, sin embargo, se ha observado que la severidad y la aparición de complicaciones es menor en este grupo. En pediatría, la mayoría de los pacientes son manejados de manera conservadora con una política de "esperar y observar" además de la implementación de medidas preventivas y de manejo sintomático. En casos específicos y evaluando de manera individual a cada paciente es posible plantear otras terapias como el uso de glucocorticoides y la cirugía descompresiva.

\section{Referencias}

1. Eha J, Pitz S, Pohlenz J. Clinical features of pediatric Graves' orbitopathy. Int Ophthalmol 2010; 30: 717-21.
2. Bartley G, Fatourechi V, Kadrmas E, Jacobsen S, Ilstrup $\mathrm{D}$, Garrity J, et al. The incidence of Graves' ophthalmopathy in Olmsted County, Minnesota. American Journal of Ophthalmology 1995; 120: 511-7.

3. Krassas G, Segni M, Wiersinga W. Childhood Graves' ophthalmopathy: results of a European questionnaire study. Eur J Endocrinol 2005; 153: 515-21.

4. Khoo D, Eng P, Ho S. Graves' ophthalmopathy in the absence of elevated free thyroxine and triiodothyronine levels: prevalence, natural history, and thyrotropin receptor antibody levels. Thyroid 2000; 10: 1093-100.

5. Wiersinga W, Smit T, van der Gaag R. Temporal relationship between onset of Graves' ophthalmopathy and onset of thyroidal Graves' disease. J Endocrinol Invest 1988; 11: 615-9.

6. Iyer S, Bahn R. Immunopathogenesis of Graves' ophthalmopathy: The role of the TSH receptor. Best Practice \& Research Clinical Endocrinology \& Metabolism 2012; 26: 281-9.

7. Prabhakar B, Bahn R, Smith T. Current perspective on the pathogenesis of Graves' disease and ophthalmopathy. Endocrine Reviews 2003; 24: 802-35.

8. Wiersinga W. TSHR and IGF-1R Autoimmunity in Graves' Ophthalmopathy J Clin Endocrinol Metab 2011; 96 (8): 2386-94.

9. Pappa A, Lawson JM, Calder V. T cells and fibroblasts in affected extraocular muscles in early and late thyroid associated ophthalmopathy. British Journal of Ophthalmology 2000; 84: 517-22.

10. Smith T, Koumas L, Gagnon A. Orbital fibroblast heterogeneity may determine the clinical presentation of thyroidassociated ophthalmopathy. J Clin Endocrinol Metab 2002; 87: 385-92.

11. Koumas L, Smith TJ, Phipps RP. Fibroblast subsets in the human orbit: Thy-1p and Thy-1-subpopulations exhibit distinct phenotypes. European Journal of Immunology 2002; 32: 477-85.

12. Khoo TK, Coenen MJ, Schiefer AR. Evidence for enhanced Thy-1 (CD90) expression in orbital fibroblasts from patients with Graves'ophthalmopathy. Thyroid 2008; 18 (12): 1291-6.

13. Hansen C, Rouhi R, Forster G, Kahaly GJ. Increased sulfation of orbital glycosaminoglycans in Graves' ophthalmopathy. J Clin Endocrinol Metab 1999; 84: 1409-13.

14. Feliciello A, Porcellini A, Ciullo C, Bonavolonta` G, Avvedimento EV, Fenzi G. Expression of thyrotropin-receptor mRNA in healthy and Graves' retro-orbital tissue. Lancet 1993; 342: 337-8.

15. Bahn RS, Dutton CM, Natt N, Joba W, Spitzweg C, Heufelder AE. Thyrotropin receptor expression in 
Graves' orbital adipose/connective tissues: potential autoantigen in Graves' ophthalmopathy. J Clin Endocrinol Metab 1998; 83: 998-1002.

16. Gerding MN, van der Meer JW, Broenink M, et al. Association of thyrotrophin receptor antibodies with the clinical features of Graves' ophthalmopathy. Clinical Endocrinology 2000; 52: 267-71.

17. Lytton SD, Ponto KA, Kanitz M. A novel thyroid stimulating immunoglobulin bioassay is a functional indicator of activity and severity of Graves' orbitopathy. Journal of Clinical Endocrinology and Metabolism 2010; 95: 2123-31.

18. Wiersinga WM. Autoimmunity in Graves' ophthalmopathy: the result of an unfortunate marriage between TSH receptors and IGF-1 receptors? J Clin Endocrinol Metab 2011; 96: 2386-94.

19. Bahn RS. Pathophysiology of Graves' ophthalmopathy: the cycle of disease. J Clin Endocrinol Metab 2003; 88: 1939-46.

20. Tsui S, Naik V, Hoa N, et al. Evidence for an association between thyroid-stimulating hormone and insulin-like growth factor 1 receptors: a tale of two antigens implicated in Graves' disease. J Immunol 2008; 181: 4397-405.

21. Stan M, Bahn R. Risk Factors for Development or Deterioration of Graves' Ophthalmopathy. Thyroid 2010; 20 (7): 777-83.

22. Bartalena L. Prevention of Graves' ophthalmopathy. Best Practice \& Research Clinical Endocrinology \& Metabolism 2012; 26: 371-9.

23. Prummel MF, Wiersinga WM. Smoking and risk of Graves' disease. Journal of the American Medical Association 1993; 269: 479-82.

24. Eckstein A, Quadbeck B, Mueller G. Impact of smoking on the response to treatment of thyroid associated ophthalmopathy. British Journal of Ophthalmology 2003; 87: 773-6.

25. Cawood TJ, Moriarty P, O'Farrelly C, O'Shea D. Smoking and thyroid-associated ophthalmopathy: a novel explanation of the biological link. J Clin Endocrinol Metab 2007; 92: 59-64.

26. Kung AW, Yau CC, Cheng A. The incidence of ophthalmopathy after radioiodine therapy for Graves' disease: prognostic factors and the role of methimazole. J Clin Endocrinol Metab 1994; 79: 542-6.

27. Bartalena L, Baldeschi L, Dickinson A. Consensus statement of the European Group on Graves' orbitopathy (EUGOGO) on management of GO. European Journal of Endocrinology 2008; 158: 273-85.

28. Traisk F, Tallstedt L, Abraham-Nordling M, et al. Thyroid-associated ophthalmopathy after treatment for Graves' hyperthyroidism with antithyroid drugs or iodi- ne-131. The J Clin Endocrinol Metab 2009; 94: 3700-7.

29. Acharya SH, Avenell A, Philip S. Radioiodine therapy (RAI) for Graves' disease (GD) and the effect on ophthalmopathy: a systematic review. Clinical Endocrinology (Oxford) 2008; 69: 943-50.

30. Lai A, Sassi L, Compri E, et al. Lower dose prednisone prevents radioiodine-associated exacerbation of initially mild or absent Graves' orbitopathy: a retrospective color study. The Journal of Clinical Endocrinology and Metabolism 2010; 95: 1333-7.

31. Uretsky SH, Kennerdell JS, Gutai JP. Graves' ophthalmopathy in childhood and adolescence. Arch Ophthalmol 1980; 98: 1963-4.

32. Gruters A. Ocular manifestations in children and adolescents with thyrotoxicosis. Exp Clin Endocrinol Diabetes 1999; 107 (5): 172-4.

33. Bartley GB, Fatourechi V, Kadramas EF. Clinical features of Graves' ophthalmopathy in an incidence cohort. Am J Ophthalmol 1996; 121: 284-90.

34. Chan W, Wong G, Fan D, Cheng A, Lam D, Ng J. Ophthalmopathy in childhood Graves'disease $\mathrm{Br} J$ Ophthalmol 2002; 86: 740-2.

35. Vikram D. Durairaj, George B. Bartley, James A. Garrity. Clinical features and treatment of Graves' ophthalmopathy in pediatric patients. Ophthalmic Plastic and Reconstructive Surgery 2006; 22 (1): 7-12.

36. Holt H, Hunter D, Smith J, Dagi L. Pediatric Graves' ophthalmopathy: The pre- and postpubertal experience. JAAPOS 2008; 12: 357-60.

37. Zanolli M, Araya A, Cattani A, Orellana P, Martínez-Aguayo A. Enfermedad de Basedow Graves en pacientes pediátricos. Rev Chil Pediatr 2008; 79: 1 .

38. Kirsch E, von Arx G, Hammer B. Imaging in Graves' orbitopathy. Orbit 2009; 28 (4): 219-25.

39. Gonçalves AC, Gebrim EM, Monteiro ML. Imaging studies for diagnosing Graves' orbitopathy and dysthyroid optic neuropathy. Clinics (Sao Paulo) 2012; 67 (11): 1327-34.

40. Müller-Forell W, Kahaly GJ. Neuroimaging of Graves' orbitopathy. Best Practice \& Research Clinical Endocrinology \& Metabolism 2012; 26: 259-71.

41. Dolman PJ. Evaluating Graves' Orbitopathy. Best Practice \& Research Clinical Endocrinology \& Metabolism 2012; 26: 229-48.

42. Dolman PJ, Rootman J. VISA classification for Graves' orbitopathy. Ophthalmic Plastic and Reconstructive Surgery 2006; 22 (5): 319-24.

43. Yeatts RP. Quality of life in patients with Graves ophthalmopathy. Trans Am Ophthalmol Soc 2005; 103: 368-411.

44. Fayers T, Dolman PJ. Validity and reliability of the 
TED-QOL: a new three-item questionnaire to assess quality of life in thyroid eye disease. British Journal of Ophthalmology 2011; 95 (12): 1670-4.

45. Bahn RS. Graves' ophthalmopathy. N Engl J Med 2010; 362: 726-38.

46. Bartalena L, Tanda ML. Graves' ophthalmopathy. N Engl J Med 2009; 360: 994-1001.

47. Marcocci C, Bartalena L, Tanda ML, et al. Comparison of the effectivenessand tolerability of intravenous or oral glucocorticoids associated with orbital radiotherapy in the management of severe Graves' ophthalmopathy: results of a prospective, single-blind, randomized study. J Clin Endocrinol Metab 2001; 86: 3562-7.

48. Zang S, Ponto KA, Kahaly GJ. Intravenous Glucocorticoids for Graves' Orbitopathy: Efficacy and Morbidity. J Clin Endocrinol Metab 2011; 96: 320-32.

49. Wei Zhu, Lei Ye, Liyun Shen, Qin Jiao, Fengjiao Huang,
Rulai Han, et al. A Prospective, Randomized Trial of Intravenous Glucocorticoids Therapy With Different Protocols for Patients With Graves' Ophthalmopathy. J Clin Endocrinol Metab 2014; 99: 1999-2007.

50. Krassas GE, Rivkees SA, Kiess W. Thyroid-Associated Ophthalmopathy in Juvenile Graves'Disease: Clinical, Endocrine and Therapeutic Aspects. Journal of Pediatric Endocrinology \& Metabolism 2006; 19: 1193-206.

51. Stiebel-Kalish H, Robenshtok E, Hasanreisoglu M, Ezrachi D, Shimon I, Leibovici L. Treatment Modalities for Graves' phthalmopathy: Systematic Review and Metaanalysis. J Clin Endocrinol Metab 2009; 94: 2708-16.

52. Marcocci C, Marinò M. Treatment of mild, moderate-to-severe and very severe Graves' orbitopathy. Best Practice \& Research Clinical Endocrinology \& Metabolism 2012; 26: 325-37. 